\title{
A BRONCHIECTATIC PATIENT'S RISK OF PNEUMONIA AND PROGNOSIS
}

\author{
Olli Säynäjäkangas ${ }^{1}$, Timo Keistinen ${ }^{2}$ \\ ${ }^{1}$ Lapland Central Hospital, Department of Pulmonary Diseases, Rovaniemi, Finland \\ ${ }^{2}$ Institute of Health Sciences, University of Oulu, and Vaasa Health Care Region, Vaasa, Finland
}

\begin{abstract}
SUMMARY
The objective of this study was to define the morbidity and mortality of bronchiectatic patients.

All records from the years 1993-2004 of patients with asthma, chronic obstructive pulmonary disease (COPD) and bronchiectasis as the primary diagnosis were extracted from the Finnish Hospital Discharge Register. The data of these patients' deaths until the end of the year 2004 were acquired from Statistics Finland. These materials were analyzed in order to find each bronchiectatic patient of this period an asthma or COPD control subject who was of the same age and sex and had also been hospitalized in the same year. Their numbers of pneumonia and prognoses were compared with each other during the study period.

$59.4 \%$ of all bronchiectasis treatment periods in absolute numbers were for people aged 65 years or over. The occurrence of pneumonia in bronchiectatic patients was $1.03(95 \% \mathrm{Cl} 0.82-1.24)$ per follow-up year, while the corresponding rate in the COPD control subjects was $1.22(95 \%$ $\mathrm{Cl} 0.92-1.53)$ and in the asthma control subjects $0.38(95 \% \mathrm{Cl} 0.22-0.54)$. The mean survival times for the bronchiectatic patients were $8.33(95 \%$ $\mathrm{Cl} 8.16-8.50)$, for the COPD control subjects $6.26(95 \% \mathrm{Cl} 6.07-6.45)$ and for the asthma patients $8.93(95 \% \mathrm{Cl} 8.76-9.10)$ years.

Bronchiectasis-related hospitalization in Finland is primarily focused on aged people. A bronchiectatic patient has a higher risk of pneumonia and a worse prognosis than an asthmatic, while the situation is opposite when compared to a COPD patient.
\end{abstract}

Key words: bronchiectasis, pneumonia, hospitalization, mortality, prognosis

Address for correspondence: O. Säynäjäkangas, Lapland Central Hospital, Department of Pulmonary Diseases, Ounasrinteentie 22, 96440 Rovaniemi, Finland. E-mail: olli.saynajakangas@|shp.fi, olli.saynajakangas@pp1.inet.fi

\section{INTRODUCTION}

While bronchiectasis remains a concern in the developing world, it is less common in developed countries. Infections are still the most common causes for it both among adults (1) and children (2). It has been estimated that COPD patients have a fourfold and asthma patients one-and-a-half-fold risk to contract pneumonia requiring hospitalization if compared to basic population (3). Mortality among COPD patients is remarkably higher than among asthmatics (4). The risk of pneumonia and the prognosis have been documented more poorly among bronchiectatic patients.

The purpose of this study was to study, by means of the Discharge Register of Hospitals and the Cause of Death Register of Statistics Finland, bronchiectasis-related hospitalization and the risk of pneumonia among these patients and its prognosis when compared to asthma and COPD patients.

\section{MATERIALS AND METHODS}

All treatment data from the year 1993 to 2004 in which the primary or secondary diagnosis was a chronic obstructive pulmonary disease (COPD, "491", "492", "496", ICD 9 during 1993-1995 and "J41-J44", ICD 10 from the year 1996 onwards), asthma ("493", ICD 9 and "J45-J46", ICD 10) and bronchiectasis
(“494”, ICD 9 and “J47”, ICD 10) were extracted from the Hospital Discharge Register maintained by the National Research and Development Centre for Welfare and Health. A total of 510,303 treatment periods of this kind were found for 159,744 patients. The trends of hospitalization of bronchiectatic patients (the disease as a primary or secondary diagnosis) were classified by age groups according to sex in proportion to population in 1993-2004. Furthermore, each bronchiectatic patient was found an asthma or COPD patient of the same sex as a control subject, who was hospitalized in the same year and whose age on the admission day was as near the bronchiectatic patient's age as possible. This age was 63.7 years in bronchiectatic patients and 64.4 years in both of the control groups. In the bronchiectasis cases, it was possible that asthma or COPD was a primary or secondary diagnosis during a treatment period, but the asthma control subjects did not have any bronchiectasis or COPD diagnoses or the COPD control subjects did not have any asthma or bronchiectasis diagnoses. A follow-up time was defined for these patients which was the time between a patient's admission date and the day on which (s)he died/31.12.2004. Their risk of pneumonia was defined by dividing the number of their pneumonia-related (codes "48*", "076", ICD 9 and "J11-J18", ICD 10) stays in hospital by the follow-up time. The difference between the numbers of pneumonias on annual level was compared in the SPSS 13.0 for Windows statistical programme with OneWay Anova. Furthermore, the 
prognoses of these three patient groups were defined from the admission day until death/31.12.2004 by using Kaplan Meyers survival analysis and Log Rank test in the same programme.

\section{RESULTS}

\section{Treatment Periods}

Diagnosis of bronchiectasis was the primary or secondary diagnosis in 8,453 treatment periods for 3,051 patients. 55.1\% of the bronchiectatic patients were women. At the beginning of bronchiectasis-related hospitalization periods, men were 63.8 years and women 64.9 years on the average (Fig. 1). Only 53 bronchiectasis treatment periods were for 32 patients aged less than 15 years during the whole study period. $59.4 \%$ of all bronchiectasis treatment periods in absolute numbers were for patients aged 65 or over. The number of bronchiectasis treatment periods decreased from 161 to 133 per million persons during the period

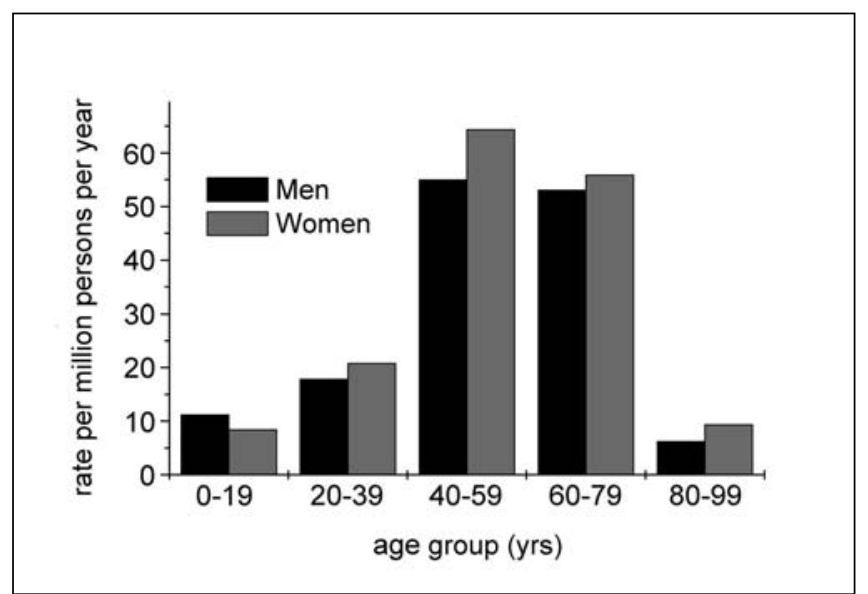

Fig. 1. Rates of bronchiectatic patients hospitalized in 1993-2004 per one million persons per year by age groups of twenty years in men (black bars) and women (grey bars).

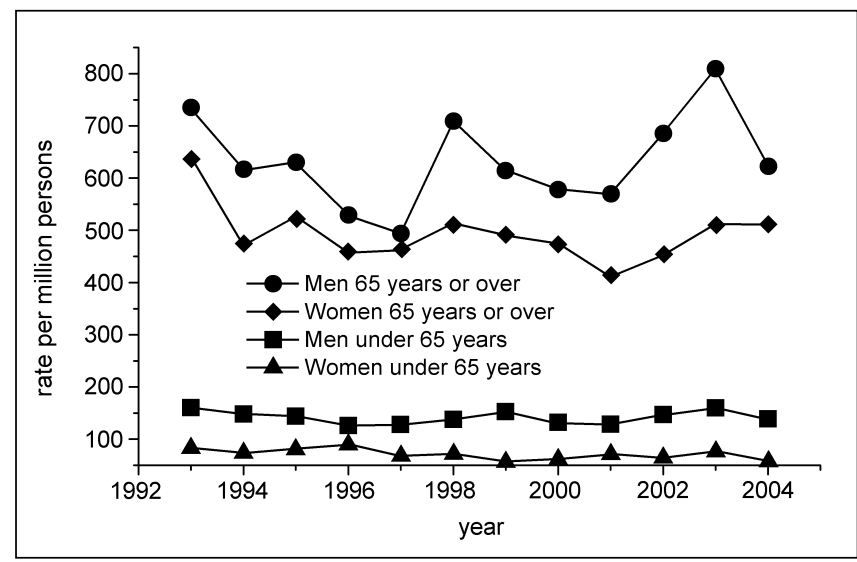

Fig. 2. Bronchiectasis-related treatment periods (the disease as a primary or secondary diagnosis) for men (dash) aged 65 years or over (circles) and under 65 years (squares) and for women (dots) aged 65 years or over (down triangles) and under 65 years (up triangles) in 1993-2004.
1993-2004. In men aged 65 or over, this decrease was 15.2\% in proportion to population and in women of the corresponding age 20.0\% (Fig. 2).

\section{Risk of Pneumonia}

Pneumonia was the primary or secondary diagnosis in $11.9 \%$ of the treatment periods of bronchiectatic patients, in $7.2 \%$ of the COPD and in $5.5 \%$ of the asthma control subjects. In the treatment periods including bronchiectasis, the follow-up time averaged 3.8 years, while in the treatment periods of the COPD control subjects it was 2.9 years and of the asthma control subjects 4.6 years. Bronchiectatic patients had pneumonia 1.03 (95\% CI 0.82-1.24) time per follow-up year on the average, while the corresponding rate in the COPD control group was 1.22 (95\% CI 0.92-1.53) and in the asthma control group 0.38 (95\% CI 0.22-0.54).

\section{Survival}

1,119 (36.7\%) of the bronchiectatic patients, 1,546 (50.7\%) of the COPD control subjects and 854 (28.0\%) of the asthma control subjects had died during the years 1993-2004.

The mean survival times for the bronchiectatic patients were 8.33 (95\% CI 8.16-8.50), for the COPD control subjects 6.26 (95\% CI 6.07-6.45) and for the asthma patients 8.93 (95\% CI 8.76-9.10) years. These differences between the bronchiectatic patients and the control groups were significant $(\mathrm{p}=0.000, \log$ rank test). The corresponding mean survival times in these patient groups were 7.84 (95\% CI 7.58-8.10), 5.84 (95\% CI 5.56-6.12) and 8.64 (95\% CI 8.39-8.90) for men and 8.73 (95\% CI 8.518.95), 6.61 (95\% CI 6.36-6.87) and 9.15 (95\% CI 8.92-9.37) years for women. Again, these differences in survival times between the bronchiectatic patients and the control groups were also significant by sexes ( $p=0.000$, Log Rank test $)$.

\section{Causes of Death}

The bronchiectatic patients had more pneumonia diagnoses (6.3\% of all the cases) as a basic cause of death than the dead asthma (5.9\%) and COPD (4.3\%) control subjects, and it was clearly the most common immediate cause of death $(22.9 \%)$ in them (Table 1). The immediate cause of death had been recorded in $53.7 \%$ of the cases.

\section{DISCUSSION}

The data of the Finnish Hospital Discharge Register are considered to be reliable. According to the study by Keskimäki (5), the dates of admission and discharge had been recorded correctly in $96 \%$ of the treatment periods and similarly the primary diagnosis in $94.1 \%$ of the respiratory diseases. Comparison of the heart and blood vessel register with this register (6) has given similar results. The asthma and COPD diagnoses of the materials can be considered quite reliable, because in the Finnish health care system it is pulmonary physician who has mainly made these diagnoses because of practice of reimbursing medicines. Similarly, the diagnosis of bronchiectasis has been based on an examination made by a specialist, on bronchography until the 
Table 1. Percentages of basic and immediate causes of death of bronchiectatic patients $(B)$ who were hospitalized and died in 1993-2004 and of asthma (A) and COPD (C) control subjects

\begin{tabular}{|l|c|c|c|}
\hline & B & A & C \\
\hline \multicolumn{4}{|l|}{ Basic cause of death } \\
\hline Pulmonary cancer & $5.3 \%$ & $5.7 \%$ & $11.91 \%$ \\
\hline Asthma & $2.0 \%$ & $4.9 \%$ & $0.2 \%$ \\
\hline COPD & $17.1 \%$ & $0.5 \%$ & $27.6 \%$ \\
\hline Pneumonia & $6.3 \%$ & $5.9 \%$ & $4.3 \%$ \\
\hline Circulatory disease & $27.8 \%$ & $49.4 \%$ & $32.1 \%$ \\
\hline Another cause & $41.6 \%$ & $33.6 \%$ & $23.9 \%$ \\
\hline Immediate cause of death & & & \\
\hline Asthma & $0 \%$ & $0.2 \%$ & $0 \%$ \\
\hline COPD & $0.7 \%$ & $0 \%$ & $0.5 \%$ \\
\hline Pneumonia & $22.9 \%$ & $12.6 \%$ & $14.9 \%$ \\
\hline Circulatory disease & $15.3 \%$ & $20.3 \%$ & $16.6 \%$ \\
\hline Another or missing cause & $61.2 \%$ & $66.9 \%$ & $67.9 \%$ \\
\hline
\end{tabular}

end of the 1980's and after that on high-resolution computed tomography (HRCT) of lungs. Changing over from version 9 to version 10 of ICD classification of diseases at the beginning of the year 1996 may have affected the results to some extent. This registry study deals with hospitalized patients only, and that is why there are more bronchiectatic patients in reality. Although these results are inpatient only, the risks found may apply to the whole community with the disease including those not yet requiring admissions for treatment. At the beginning of the study period, there were 5.1 million inhabitants in Finland, at the end of the period 5.2 million.

There has not been an essential decrease in bronchiectasis-related hospitalization during the last ten years in Finland. In their childhood in the 1940's and 1950's, the large age groups suffered

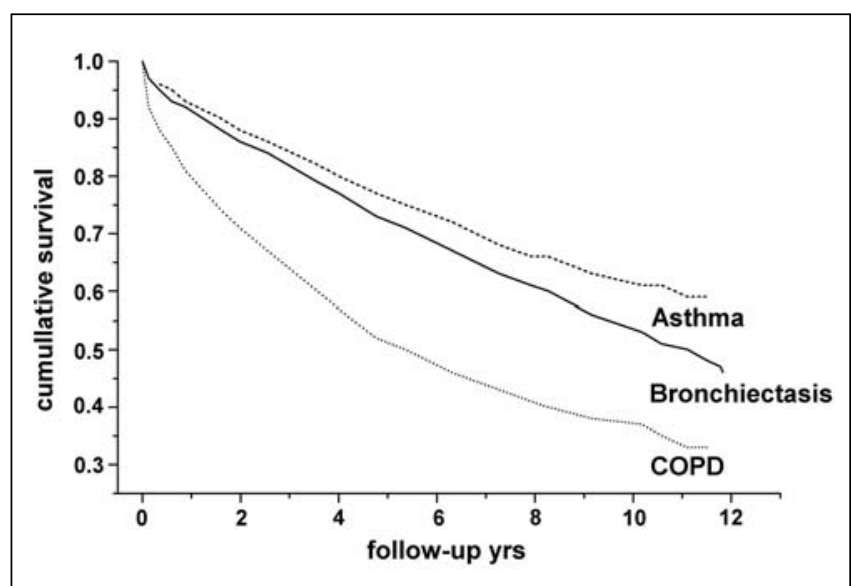

Fig. 3. Cumulative survival of bronchiectatic patients (solid line), asthma control subjects (dash) and COPD control subjects (dot) hospitalized during the years 1993-2004. from lung infections, which will still burden our public health services for a long time because of bronchiectasis. However, there has been a remarkable decrease in cases of tuberculosis in Finland during the last few decades. From the year 1960 to 1993, the annual number of reported new cases of tuberculosis in Finland decreased from 172 to 10 cases per 100,000 persons. Cases of respiratory organ tuberculosis accounted for $70 \%$ of all these cases (7). Instead, tuberculosis in children has decreased because of effective vaccination programmes and optimal treatment of infections. Cystic fibrosis, one important cause in bronchiectasis, is rare in Finland. In 2005, there were only 64 patients suffering from this disease and there was one case per about 25,000-30,000 born children in the whole country. In developing countries, bronchiectasis is still a noteworthy disease and it will probably be one until the distant future because of poor social conditions and scanty health care resources (8). In Alaska native population, prevalence of bronchiectasis in children is still high, which is thought to be associated with high pneumonia rates among children (9). On the other hand, in a developed country like New Zealand, more cases of children's bronchiectasis have been reported than in Finland, for example (10).

Bronchiectasis is often associated with a chronic obstructive pulmonary disease (11) and vice versa (12). In bronchiectasis, anatomical damages of bronchi are usually confined to certain parts of lungs, while in chronic bronchitis changes are extensive and usually associated with prolonged smoking. It is true that one patient can have both of them, which can be seen in these materials as well, and which may have partly affected the results. Similarly, if children with asthma have a bad response to inhalation steroid, there may be bronchiectasis in the background (13). As many as three of four Alaska native children with bronchiectasis had asthma (7), while on the other hand about one fifth of hospitalized bronchiectatic patients had diagnosis of asthma, too (14).

The bronchiectatic patients in our findings were hospitalized and it is an obvious fact that for this reason they were ten years older than the patients in Pasteur's publications (1), i.e. just under or over 65 years. On the other hand, hospitalization is getting rare among very old bronchiectatic patients, which can be explained by the effect of the disease making a patient's prognosis worse. More than one half of the patients were women, which conforms to earlier studies $(11,15)$. On the other hand, in our study men were more often treated in hospitals than women of the same age in relation to the population. One logical explanation for this phenomen may be that by our research men had more often the combination of bronchiectasis and COPD predisposing to hospitalization.

In our results, the bronchiectatic patients had a higher than twofold risk of contracting pneumonia if compared to asthma patients. Bronchiectatic patients had percentually more treatment periods including pneumonias than COPD patients, but the latter group's risk of pneumonia per time unit was higher because of their shorter follow-up time arising from worse prognosis. Bronchiectasis is often preceded by pneumonia (9) and damaged bronchi are easily colonised with bacteria, which adds the possibility of infections. Hospitalized child patients often have both asthma and pneumonia diagnoses (16).

The results of the prognosis of bronchiectasis conformed to our earlier study which dealt with new bronchiectasis treatment periods during the years 1982-1992 (17). In the materials presented 
now, pneumonia was more marked as a basic and especially as an immediate cause of death of bronchiectatic patients, if compared to asthma and COPD patients (Fig. 3). In the middle of the study period, women's average expected lifetime in Finland was eight years longer than men's, which was reflected as women's better prognoses both among bronchiectatic patients and control subjects.

\section{CONCLUSIONS}

Aged people are still hospitalized because of bronchiectasis unlike children among whom it is very rare in Finland. A hospitalized bronchiectatic patient has a higher risk of contracting pneumonia and a worse prognosis than an asthmatic, while the situation is reverse if compared to a COPD patient.

Conflict of Interest Statement: None of the authors have a conflict of interest to declare in relation to this work. Ethical approval: This study has been approved by the Finnish data register authorities.

\section{REFERENCES}

1. Pasteur MC, Helliwell SM, Houghton SJ, Webb SC, Foweraker JE, Coulden RA, et al. An investigation into causative factors in patients with bronchiectasis. Am J Respir Crit Care Med. 2000 Oct;162(4 Pt 1):1277-84.

2. Karakoc GB, Yilmaz M, Altintas DU, Kendirli SG. Bronchiectasis: still a problem. Pediatr Pulmonol. 2001 Aug;32(2):175-8.

3. Lee TA, Weaver FM, Weiss KB. Impact of pneumococcal vaccination on pneumonia rates in patients with COPD and asthma. J Gen Intern Med. 2007 Jan;22(1):62-7.

4. Jacobson L, Hertzman P, Löfdahl CG, Skoogh BE, Lindgren B. The economic impact of asthma and chronic obstructive pulmonary disease (COPD) in Sweden in 1980 and 1991. Respir Med. 2000 Mar;94(3):24755.
5. Keskimäki I, Aro S. Accuracy of data on diagnoses, procedures and accidents in the Finnish Hospital Discharge Register. Int J Health Sci. 1991;2:5-21.

6. Pajunen P, Koukkunen H, Ketonen M, Jerkkola T, Immonen-Räihä P, Kärjä-Koskenkari P, et al. The validity of the Finnish Hospital Discharge Register and Causes of Death Register data on coronary heart disease. Eur J Cardiovasc Prev Rehabil. 2005 Apr;12(2):132-7.

7. Tala E, Viljanen M. Mycobacterial infections in Finland. Scand J Infect Dis Suppl. 1995;98:7-8.

8. Adebonojo SA, Grillo IA, Osinowo O, Adebo OA. Suppurative diseases of the lung and pleura: a continuing challenge in developing countries. Ann Thorac Surg. 1982 Jan;33(1):40-7.

9. Singleton R, Morris A, Redding G, Poll J, Holck P, Martinez P, et al. Bronchiectasis in Alaska Native children: causes and clinical courses. Pediatr Pulmonol. 2000 Mar;29(3):182-7.

10. Twiss J, Metcalfe R, Edwards E, Byrnes C. New Zealand national incidence of bronchiectasis "too high" for a developed country. Arch Dis Child. 2005 Jul;90(7):737-40.

11. King PT, Holdsworth SR, Freezer NJ, Villanueva E, Holmes PW. Characterisation of the onset and presenting clinical features of adult bronchiectasis. Respir Med. 2006 Dec;100(12):2183-9.

12. Patel IS, Vlahos I, Wilkinson TM, Lloyd-Owen SJ, Donaldson GC, Wilks M, et al. Bronchiectasis, exacerbation indices, and inflammation in chronic obstructive pulmonary disease. Am J Respir Crit Care Med. 2004 Aug 15;170(4):400-7.

13. Nuhođlu Y, Bahçeciler N, Yüksel M, Kodalli N, Barlan IB, Yildizeli $\mathrm{B}$, et al. Thorax high resolution computerized tomography findings in asthmatic children with unusual clinical manifestations. Ann Allergy Asthma Immunol. 1999 Mar;82(3):311-4.

14. Säynäjäkangas $\mathrm{O}$, Keistinen T, Tuuponen T, Kivelä SL. Links between hospital diagnoses of bronchiectasis and asthma. Allergy. 1997 Nov;52(11):1120-2

15. Nicotra MB, Rivera M, Dale AM, Shepherd R, Carter R. Clinical, pathophysiologic, and microbiologic characterization of bronchiectasis in an aging cohort. Chest. 1995 Oct;108(4):955-61.

16. Bundy DG. Hospitalizations with primary versus secondary discharge diagnoses of asthma: implications for pediatric asthma surveillance. $\mathrm{J}$ Pediatr. 2007 Apr;150(4):446-9.

17. Keistinen T, Säynäjäkangas O, Tuuponen T, Kivelä SL. Bronchiectasis: an orphan disease with a poorly-understood prognosis. Eur Respir J. 1997 Dec;10(12):2784-7. 